Pacific Journal of Mathematics

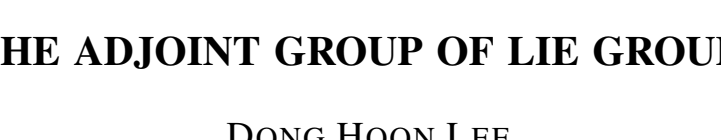




\title{
THE ADJOINT GROUP OF LIE GROUPS
}

\author{
DoNG HoON LEE
}

Let $G$ be a Lie group and let $\operatorname{Aut}(G)$ denote the group of automorphisms of $G$. If the subgroup $\operatorname{Int}(G)$ of innerautomorphisms of $G$ is closed in $\operatorname{Aut}(G)$, then we call $G$ a $(C A)$ group (after Van Est.). In this note, we investigate $(C A)$ property of certain classes of Lie groups. The main results are as follows:

Theorem A. Let $G$ be an analytic group and suppose that there is no compact semisimple normal subgroup of $G$. If $G$ contains a closed uniform $(C A)$ subgroup $H$, then $G$ is $(C A)$.

Theorem B. If $G$ is an analytic group whose exponential map is surjective, then $G$ is $(C A)$.

In [3], Garland and Goto proved that if an analytic group $G$ contains a lattice, then $G$ is $(C A)$. Since a lattice in a solvable group is a uniform lattice, it is finitely generated and so the automorphism group of this uniform lattice is discrete, and thus this lattice is trivially a $(C A)$ subgroup. Thus Theorem A generalizes the above theorem of Garland and Goto for solvable groups. Theorem B is an improvement of the well known theorem that every nilpotent analytic group is $(C A)$ (see [2]). In $\S 1$, we introduce some notation and preliminary materials. $\S 2$ and $\S 3$ are devoted for the proofs of the main theorems together with their immediate corollaries.

1. Preliminaries and notations. The group $\operatorname{Aut}(G)$ of automorphisms of locally compact a topological group $G$ may be regarded as a topological group, the topology being the (generalized) compact open topology defined as in [5]. Thus, if we denote by $N(C, V)$ the set of all $\theta \in \operatorname{Aut}(G)$ for which $\theta(x) x^{-1} \in V$ and $\theta^{-1}(x) x^{-1} \in V$ whenever $x \in C$, then the sets $N(C, V)$ form a fundamental system of neighborhoods of the identity element of $\operatorname{Aut}(G)$ as $C$ ranges over the compact subsets of $G$ and $V$ over the set of neighborhoods of the identity element of $G$.

If $G$ is an analytic group and $\mathscr{G}$ its Lie algebra, then $\operatorname{Aut}(G)$ may be identified with a closed subgroup of the linear group Aut(C) of automorphisms of $\mathscr{G}$. Under this identification, $\operatorname{Int}(G)$ coincides with the adjoint group $\operatorname{Int}(\mathscr{G})$, which is generated by $e^{a d X}, X \in \mathscr{G}$ where $a d$ denotes the adjoint representation of $\mathscr{G}$. Thus the $(C A)$ property of analytic groups are entirely determined by their Lie algebras. In particular, if $\widetilde{G}$ is a covering group of $G$ and if $G$ is $(C A)$, then so is $\widetilde{G}$. This fact is used in the proofs of the main theorems. 
Throughout this paper the following notation is used: If $A$ is a subgroup of $G$, then $\operatorname{Int}_{G}(A)$ denotes the subgroup of $\operatorname{Int}(G)$ which consists of inner automorphisms induced by elements of $A$. Thus $\operatorname{Int}_{G}(G)$ is merely equal to $\operatorname{Int}(G)$. The center of $G$ is denoted with $Z(G)$. Also if $x \in G$, then $I_{x}$ means the inner automorphism induced by $x$.

2. Proof of Theorem A. Let $H$ be a closed uniform subgroup of an analytic group $G$, and $H_{0}$ its identity component. Then $H / H_{0}$ is finitely generated. In order to see this, let $\widetilde{G}$ be the simply connected covering group of $G, \widetilde{H}$ the complete inverse image of $H$ under the covering projection and $\widetilde{H}_{0}$ the identity component of $\widetilde{H}$. Then since the covering projection induces an epimorphism $\widetilde{H} / \widetilde{H}_{0} \rightarrow H / H_{0}$, it suffices to show that $\widetilde{H} / \widetilde{H}_{0}$ is finitely generated. Nothing that $\widetilde{G} / \widetilde{H}_{0}$ is simply connected (see, for example, Mostow [7], Corollary 1, p. 617), we can identify the discrete group $\widetilde{H} / \widetilde{H}_{0}$ with the fundamental group of the compact manifold $\widetilde{G} / \widetilde{H}$. As the fundamental group of a compact manifold is finitely presented, it follows that $\widetilde{H} / \widetilde{H}_{0}$ is, in particular, finitely generated.

Now we can apply a theorem of Hochschild ([5], Th. 2, p. 212) to see that if $H$ is a closed subgroup of an analytic group, then $\operatorname{Aut}(H)$ is a Lie group.

The following lemma enables us to assume that $G$ is simply connected.

Lemma. Let $\widetilde{H}$ be a compactly generated Lie group and $A$ a closed discrete central subgroup of $\tilde{H}$. Let $H=\tilde{H} / A$. If $H$ is a $(C A)$ group, then so is $\tilde{H}$. In fact, $\operatorname{Int}(\widetilde{H})$ is a topological extension of a discrete group by $\operatorname{Int}(H)$.

Proof. Let $\pi: \widetilde{H} \rightarrow \widetilde{H} / A=H$ be the natural map and define $\chi$ : $\operatorname{Int}(\widetilde{H}) \rightarrow \operatorname{Int}(H)$ by $\chi\left(I_{\tilde{h}}\right)=I_{\pi(\tilde{h})}$, for $\tilde{h} \in \tilde{H}$

(i) $\chi$ is continuous. To see this, note first that we can find a compact nighborhood $\widetilde{D}$ of 1 in $\widetilde{H}$ which generates $\widetilde{H}$. Now let $C$ be a compact subset of $H$ and $U$ a neighborhood of 1 in $H$. Then we have to find a compact subset $\widetilde{C}$ of $\widetilde{H}$ and a neighborhood $\widetilde{U}$ of 1 in $\widetilde{H}$ so that $\chi(N(\widetilde{C}, \widetilde{U}) \cap \operatorname{Int}(\widetilde{H})) \subseteq N(C, U) \cap \operatorname{Int}(H)$. Since $\pi(\widetilde{D})=D$ is also a compact neighborhood of 1 which generates $H$, we can find a positive integer $k$ such that $C \subset D^{k}$ by using the compactness of $C$. Now letting $\widetilde{C}=\widetilde{D}^{k}$ and $\widetilde{U}=\pi^{-1}(U)$, it is easy to see that $(\widetilde{C}, \widetilde{U})$ is a desired pair. Hence $\chi$ is cotinuous.

(ii) $\chi$ is open. In fact, since $H$ is $(C A)$, the canonical map $H / Z(H) \rightarrow \operatorname{Int}(H)$ is an isomorpuism of topological groups. Hence (ii) follows from the following commutative diagram 


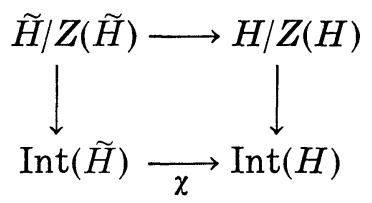

where the left vertical map is always continuous and the top one is open.

(iii) The kernel $\mathscr{K}$ of $\chi$ is a discrete subgroup of $\operatorname{Aut}(\widetilde{H})$ and hence is closed in $\operatorname{Aut}(\widetilde{H})$. To see this let $\widetilde{\mathscr{K}}$ be the closure of $\mathscr{K}$ in $\operatorname{Aut}(\widetilde{H})$, and $\overline{\mathscr{K}}_{0}$ the identity component of $\overline{\mathscr{K}}$. Since $\operatorname{Aut}(\widetilde{H})$ is a Lie group $\overline{\mathscr{K}} \mid \overline{\mathscr{K}_{0}}$ is discrete.

Since $\mathscr{K}=\operatorname{Ker}(\chi)$ and since $A$ is central in $\widetilde{H}$ every element of $\mathscr{K}$ induces the identity map on $H=\widetilde{H} / A$ and on $A$. Hence $\theta \in \overline{\mathscr{K}}$ implies that $\theta=1$ on $A$ and $\theta=1$ on $H=\widetilde{H} / A$, which implies that $\theta(\widetilde{h}) \widetilde{h}^{-1} \in A$ for $\widetilde{h} \in \widetilde{H}$.

Let $\tilde{h} \in \widetilde{H}$ be arbitrary and define $\eta_{\tilde{h}}: \overline{\mathscr{K}} \rightarrow A$ by $\eta_{\tilde{h}}(\theta)=\theta(\widetilde{h}) \widetilde{h}^{-1}$, $\theta \in \overline{\mathscr{K}}$. Then $\eta_{\tilde{h}}$ is continuous and thus $\eta_{\tilde{h}}\left(\overline{\mathscr{K}_{0}}\right)$ is connected in the discrete $A$. Since $\eta_{\tilde{h}}\left(\overline{\mathscr{K}_{0}}\right)$ contains $1, \eta_{\tilde{h}}\left(\overline{\mathscr{K}_{0}}\right)=1$ and this then implies that $\theta(\tilde{h})=\tilde{h}$ for all $\theta \in \overline{\mathscr{K}}_{0}$. Since $\tilde{h}$ is arbitrary, $\overline{\mathscr{K}}_{0}=1$ and $\overline{\mathscr{K}}$ is discrete. We have thus shown that $\mathscr{K}$ is a discrete subgroup of $\operatorname{Aut}(H)$ and hence $\mathscr{K}$ is closed in $\operatorname{Aut}(\tilde{H})$.

(iv) Since $(\operatorname{Int}(\widetilde{H}) / \mathscr{K} \cong \operatorname{Int}(H), \operatorname{Int}(\widetilde{H})$ is closed in $\operatorname{Aut}(\widetilde{H})$ as a locally compact subgroup of $\operatorname{Aut}(\widetilde{H})$ and the lemma is proved.

Now we are ready to present the proof of Theorem A. Let $\widetilde{G}$ denote the simply connected covering group of $G$ and let $\pi$ be the covering homomorphism.

Then, by the lemma $\pi^{-1}(H)=\widetilde{H}$ is also uniform and $(C A)$. Hence no generality will be lost in assuming that $G$ is simply connected. By the assumption, $\operatorname{Int}(H)$ is closed in $\operatorname{Aut}(H)$. Thus the canonical map $H / Z(H) \rightarrow \operatorname{Int}(H)$ is an isomorphism of topological groups. Define $\varphi$ : $\operatorname{Int}_{G}(H) \rightarrow \operatorname{Int}(H)$ to be the restricting homomorphism and let $\mathscr{C}$ be the closure of the kernel of $\varphi$, the closure being taken in $\operatorname{Aut}(G)$. Then $\operatorname{Int}_{G}(H) \mathscr{K}$ is a subgroup of $\operatorname{Aut}(G)$. We define $H / Z(H) \rightarrow$ $\operatorname{Int}_{G}(H) \mathscr{K} / \mathscr{K}$ and $\operatorname{Int}_{G}(H) \mathscr{K} / \mathscr{K} \rightarrow \operatorname{Int}(H)$ to be the homomorphisms induced by the canonical maps $H \rightarrow \operatorname{Int}_{G}(H)$ and $\operatorname{Int}_{G}(H) \rightarrow \operatorname{Int}(H)$, respectively. Then the following diagram commutes:

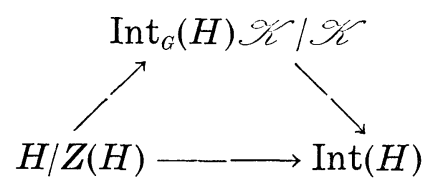

and all three maps are continuous and algebraically isomorphisms. Since the bottom one is an isomorphism of topological groups, $\operatorname{Int}(H)$ 
is topologically isomorphic with $\operatorname{Int}_{G}(H) \mathscr{K} / \mathscr{K}$ and thus the latter is a locally compact subspace of the quotient space $\operatorname{Aut}(G) / \mathscr{K}$. Hence it is closed in $\operatorname{Aut}(G) / \mathscr{K}$ and, accordingly, $\operatorname{Int}_{G}(H) \mathscr{K}$ is closed in $\operatorname{Aut}(G)$.

We next claim that $\mathscr{K} \subseteq \operatorname{Int}(G)$. In fact, if $\theta \in \mathscr{K}$, define $P(g)=$ $\theta(g) g^{-1}$, for $g \in G$. Then $P: G \rightarrow G$ is continuous, and $P(H)=\{1\}$ and $G / H$ compact imply that $P(G)$ is compact. Thus we see that $\theta$ is an automorphism of bounded displacement in the sense of Tits [8] and $\theta$ is therefore an inner automorphism induced by a central element of the nilradical of $G$ ([8], Lemma (6), p. 102). Thus $\mathscr{C} \subseteq \operatorname{Int}(G)$.

By what we have shown, it is clear now that the closure $\overline{\operatorname{Int}_{G}(H)}$ of $\operatorname{Int}_{G}(H)$ is contained in $\operatorname{Int}(G)$. Since $G / H$ is compact and since $G / H \rightarrow \operatorname{Int}(G) / \overline{\operatorname{Int}_{G}(H)}$ is continuous, $\operatorname{Int}(G)$ is compact, modulo $\overline{\operatorname{Int}_{G}(H)}$ and hence $\operatorname{Int}(G)$ is closed, proving that $G$ is $(C A)$.

COROLLARY. If a solvable analytic group $G$ contains a closed abelian uniform subgroup, then $G$ is a $(C A)$ group.

Corollary. (See, Garland and Goto [3]). If a solvable analytic group $G$ contains a lattice, then $G$ is a $(C A)$ group.

REMARK. In [6], we have shown that any extension of a simply connected $(C A)$ group by a compact connected group is a $(C A)$ group. Thus Theorem A generalizes this for the solvable case.

REMARK. We have failed to see whether or not the nonexistence of compact semi-simple normal subgroup in the theorem is necessary. This was needed in order to apply the result of Tits in the proof.

3. Proof of Theorem B. In order to prove Theorem B, we first note that an analytic group $G$ is $(C A)$ if and only if its radical is $(C A)$ (See Van Est [2]). Thus we may assume that the group in the theorem is solvable.

Let $\mathscr{G}$ be a finite-dimensional real solvable Lie algebra and let $G$ be an analytic group with its Lie algebra $\mathscr{G}$. If an exponential map exp: $\mathscr{G} \rightarrow G$ is surjective, then the exponential map into its simply connected covering group is a bijection. Thus by the remark in $\S 1$, it suffices to prove:

Theorem $\mathrm{B}^{\prime}$. Let $\mathscr{G}$ be a finite-dimensional real solvable Lie algebra. If the exponential map is a bijection, then $\mathscr{G}$ is a $(C A)$ Lie algebra (that is, the adjoint group $\operatorname{Int}(\mathscr{G})$ is closed in $\operatorname{Aut}(\mathscr{G})$ ).

In order to prove this, we need the following lemma: 
Lemma. Let $\mathscr{N}$ be the nilradical of $\mathscr{G}$. If $X \in \mathscr{N}$ then the one-parameter subgroup $\left\{e^{a d(t X)}: t \in R\right\}$ is closed in $\operatorname{Aut}(\mathscr{G})$.

Proof of lemma. Let $T$ denote the given one-parameter subgroup. We show if $T$ is not closed, then $T$ is trivial. In fact, if $T$ is not closed in $\operatorname{Aut}(\mathscr{G})$, then the closure $\bar{T}$ of $T$ is compact. Define $\varphi$ : $\operatorname{Aut}(\mathscr{G}) \rightarrow \operatorname{Aut}(\mathscr{N})$ to be the restricting homomorphism. Since $\mathscr{N}$ is a characteristic ideal in $\mathscr{G}, \varphi$ is well defined and is continuous.

Now let $a d_{\mathscr{r}}$ denote the adjoint representation of the nilpotent Lie algebra $\mathscr{N}$. Since $\mathscr{N}$ is nilpotent, $\operatorname{Int}(\mathscr{N})$ is closed in $\operatorname{Aut}(\mathscr{N})$ ([2], Proposition 1.2.2, p. 322), and thus $\overline{\varphi(T)} \subset \operatorname{Int}(\mathscr{N})$. By using the fact that the maximal compact subgroup of any nilpotent analytic group is contained in its center, it follows that $\operatorname{Int}(\mathscr{N})$ is always simply connected. Hence the compact subgroup $\overline{\varphi(T)}$ must be trivial, which means that $a d_{\mathscr{S}} X=0$ and so $X$ is central in $\mathscr{N}$.

Next we show that $X$ is central in $\mathscr{C}$. In order to see this, note first that $[X, \mathscr{G}] \subseteq \mathscr{G}^{\prime} \subseteq \mathscr{N}, \mathscr{G}^{\prime}$ being the commutator subalgebra of $\mathscr{G}$. Thus $X$ being a central element of $\mathscr{N}$ implies that $\operatorname{ad}(X)^{2}=0$. Therefore $e^{a d(t X)}=1+a d(t X)$ for $\mathrm{t} \in R$. Let $Y \in \mathscr{G}$ be arbitrary. Thus we have

$$
\begin{aligned}
\exp (R[X, Y]) & =\exp (\operatorname{ad}(R X)(Y))=\exp \left(e^{a d(R X)}-1\right)(Y) \\
& =\exp ((T-1)(Y)) .
\end{aligned}
$$

Since $\bar{T}$ is compact, the closure of $T-1$ is compact in the matrix topology of $\operatorname{End}(\mathscr{G})$, the ring of endomorphisms of the vector space $\mathscr{C}$. Therefore, the continuity of exp implies that $\exp ((T-1)(Y))$ is bounded in $G$. Consequently, the one-parameter subgroup exp $(R[X, Y])$ is relatively compact. But $G$ is simply connected and thus this oneparameter subgroup must be trivial, which implies that $\operatorname{ad}(X)=0$ and we have proved that $X$ is central in ${ }^{\text {. Therere }} T=1$ as desired.

Proof of Theorem B'. By a theorem of Goto ([4], Theorem III, p. 165), it suffices to show that every one-parameter subgroup of $\operatorname{Int}(\mathscr{G})$ is closed in $\operatorname{Aut}(\mathscr{G})$. Noting that every one-parameter subgroup of $\operatorname{Int}(\mathscr{G})$ is of the form $e^{a d(R X)}$ for some $X \in \mathscr{G}$, assume that there is a nonzero $X$ such that $T=e^{\operatorname{ad}(R X)}$ is not closed in Aut( $\left.\mathscr{G}\right)$. We see from the lemma that $X$ is not in $\mathscr{N}$.

Next we select a decreasing sequence of ideals of $\mathscr{G}$ :

$$
\mathscr{G}_{0}=\mathscr{G}>\mathscr{G}_{1}>\mathscr{G}_{2}>\cdots>\mathscr{O}_{n+1}=(0)
$$

such that $\operatorname{dim}_{R}\left(\left(\mathscr{G}_{i} / \mathscr{G}_{i+1}\right) \leqq 2\right.$. Let $A_{i}$ denote the endomorphism on $\mathscr{G}_{i} / \mathscr{G}_{i+1}$ which is induced by $a d(X), i=0,1, \cdots, n$. Then there exists 
$p$ such that $A_{p} \neq 0$. For, if $A_{i}=0$ for all $i$, then $a d(X)$ would be a nilpotent transformation and hence $X \in \mathscr{N}$, which is impossible. Since $T$ is relatively compact in $\operatorname{Aut}(\mathscr{G})$, so is $S=e^{R A p}$ in $\operatorname{Aut}\left(\mathscr{G}_{p} / \mathscr{G}_{p+1}\right)$. Since $A_{p}$ is nonzero, $S$ is nontrivial and thus $\operatorname{dim}_{R}\left(\mathscr{G}_{p} / \mathscr{G}_{p+1}\right)=2$. Since a maximal compact subgroup of $\operatorname{Aut}\left(\mathscr{G}_{p} / \mathscr{G}_{p+1}\right)$ is a circle group, it follows that $S$ is a circle group in $\operatorname{Aut}\left(\mathscr{G}_{p} / \mathscr{G}_{p+1}\right)$. Now let $\pi: \mathscr{G} \rightarrow \mathscr{G} / \mathscr{G}_{p+1}$ be the natural homomorphism and let $\mathscr{H}$ be the sub-algebra of $\mathscr{G} \mid \mathscr{G}_{p+1}$ which is generated by $\pi(X)$ and $\mathscr{G}_{p} / \mathscr{G}_{p+1}$. Then from what we have seen above, it is easy to see that $\mathscr{H}$ is the Lie algebra of the group of the rigid motions on the plane. Thus exp is not a bijection by the well known theorem of Dixmier ([1], Th. 3, p. 120). Hence every oneparameter subgroup of $\operatorname{Int}(\mathscr{C})$ is closed in $\operatorname{Aut}(\mathscr{G})$, which proves the Theorem $\mathrm{B}^{\prime}$.

In the proof of Theorem $\mathrm{B}^{\prime}$, we have actually shown that $\operatorname{Int}(\mathscr{G})$ contains no compact subgroups. Hence we have:

CoRollary. Let $G$ be a solvable analytic group such that the exponential map is surjective. Then $\operatorname{Int}(G)$ is simply connected.

Corollary. Let $G$ be as above. Then $Z(G)$ is connected.

Proof. By Theorem B, $G / Z(G)=\operatorname{Int}(G)$ is an isomorphism of topological groups. Since $\operatorname{Int}(G)$ is simply connected, it follows that $Z(G)$ is connected.

REMARK. The coverse of the Theorem B is false. The group of rigid motions on the plane is perhaps the simplest example.

\section{BIBLIOGRAPHY}

1. J. Dixmier, L'application exponentielle dans les groupes de Lie résolubles, Bull. Soc Mathe France 85 (1957), 113-121.

2. W. T. van Est, Some theorems on CA Lie algebrs, Proc. Kon. Ned. Akad. Wetensch. Series A (1952), 558-568.

3. H. Garland and M. Goto, Lattices and the adjoint group of a Lie group, Trans. Amer. Math. Soc. 124 (1966), 450-460.

4. M. Goto, Dense embeddings of locally compact connected groups, Ann. of Math. 61 (1955), 154-169.

5. G. Hochschild, The automorphism group of a Lie group, Trans. Amer. Math. Soc. 72 (1952), 209-216.

6. D. H. Lee and T. S. Wu, On (CA) topological groups (to appear in Duke Math. J.)

7. G. D. Mostow, Extensibility of local Lie groups of transformations and groups on surfaces, Ann. of Math. 52 (1950), 606-638.

8. J. Tits, Automorphismes à déplacement borné des groupes de Lie, Topology 3 (1964), $97-107$.

Received July 28, 1969. This research was supported in part by the National Science Foundation Grant GP 12261. 


\section{PACIFIC JOURNAL OF MATHEMATICS}

\section{EDITORS}

\author{
H. SAMELSON \\ Stanford University \\ Stanford, California 94305 \\ Richard Pierce \\ University of Washington \\ Seattle, Washington 98105
}

J. DUGUNDJI

Department of Mathematics

University of Southern California

Los Angeles, California 90007

BASIL GORDON*

University of California

Los Angeles, California 90024

\section{ASSOCIATE EDITORS}
E. F. BECKENBACH
B. H. NeUmanN
F. WOLF
K. YoSHIDA

\section{SUPPORTING INSTITUTIONS}

UNIVERSITY OF BRITISH COLUMBIA CALIFORNIA INSTITUTE OF TECHNOLOGY

UNIVERSITY OF CALIFORNIA MONTANA STATE UNIVERSITY

UNIVERSITY OF NEVADA

NEW MEXICO STATE UNIVERSITY

OREGON STATE UNIVERSITY

UNIVERSITY OF OREGON

OSAKA UNIVERSITY

UNIVERSITY OF SOUTHERN CALIFORNIA
STANFORD UNIVERSITY

UNIVERSITY OF TOKYO

UNIVERSITY OF UTAH

WASHINGTON STATE UNIVERSITY

UNIVERSITY OF WASHINGTON

AMERICAN MATHEMATICAL SOCIETY CHEVRON RESEARCH CORPORATION TRW SYSTEMS

NAVAL WEAPONS CENTER 


\section{Pacific Journal of Mathematics}

\section{Vol. 32, No. $1 \quad$ January, 1970}

Robert Alexander Adams, Compact Sobolev imbeddings for unbounded domains ........................................ 1

Bernhard Amberg, Groups with maximum conditions .................. 9

Tom M. (Mike) Apostol, Möbius functions of order k............... 21

Stefan Bergman, On an initial value problem in the theory of two-dimensional transonic flow patterns ................... 29

Geoffrey David Downs Creede, Concerning semi-stratifiable spaces ...... 47

Edmond Dale Dixon, Matric polynomials which are higher

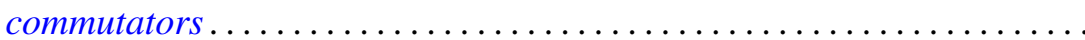

R. L. Duncan, Some continuity properties of the Schnirelmann density.

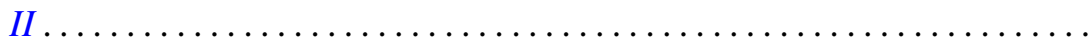

Peter Larkin Duren and Allen Lowell Shields, Coefficient multipliers of $H^{p}$

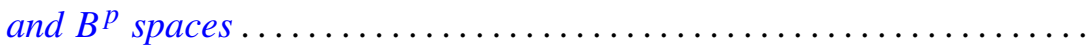

Hector O. Fattorini, On a class of differential equations for vector-valued

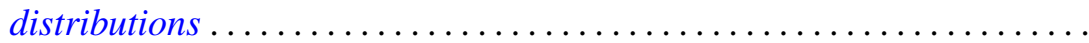

Charles Hallahan, Stability theorems for Lie algebras of derivations. . . . . . 105

Heinz Helfenstein, Local isometries of flat tori ................ 113

Gerald J. Janusz, Some remarks on Clifford's theorem and the Schur

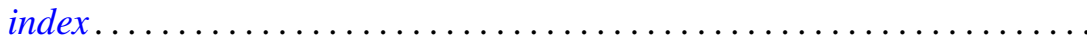

Joe W. Jenkins, Symmetry and nonsymmetry in the group algebras of discrete groups. ...

Herbert Frederick Kreimer, Jr., Outer Galois theory for separable

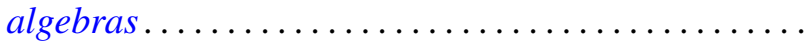

D. G. Larman and P. Mani, On visual hulls

R. Robert Laxton, On groups of linear recurrences. II. Elements of finite order.

Dong Hoon Lee, The adjoint group of Lie groups ...

James B. Lucke, Commutativity in locally compact rings

Charles Harris Scanlon, Rings of functions with certain Lipschitz

$$
\text { properties ............................... }
$$

Binyamin Schwarz, Totally positive differential systems .

James McLean Sloss, The bending of space curves into piecewise helical curves.

James D. Stafney, Analytic interpolation of certain multiplier spaces ...

Patrick Noble Stewart, Semi-simple radical classes.......

Hiroyuki Tachikawa, On left $\mathrm{QF}-3$ rings ...................... 255

Glenn Francis Webb, Product integral representation of time dependent nonlinear evolution equations in Banach spaces.... . . . 